\title{
COBRE: PRODUÇÃO INDUSTRIAL E APLICAÇÕES
}

\author{
Luciana A. Silva ${ }^{\mathrm{a}, \mathrm{b}, \#}$, Mauricio M. Victor ${ }^{\mathrm{a}, \mathrm{b}}$, Wilson Araujo Lopes $^{\mathrm{a}, \mathrm{b}}$ e Silvio Cunha ${ }^{\mathrm{a}, \mathrm{b}, *,(\mathbb{D})}$ \\ anstituto de Química, Universidade Federal da Bahia, 40170-115 Salvador - BA, Brasil

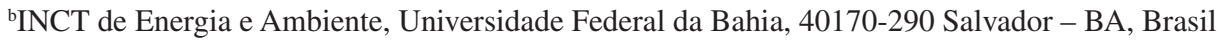

Recebido em 13/06/2019; aceito em 22/08/2019; publicado na web em 13/11/2019

\begin{abstract}
COPPER: INDUSTRIAL PRODUCTION AND APPLICATIONS. This article is dedicated to element copper in honor to International Year of the Periodic Table celebrated in 2019. Copper is one of the oldest discovered chemical elements and manipulated by human being. In this sense, aspects about the element copper and its importance in different areas are described, from its mineral exploration through its processing and applications. Inorganic aspects such as structure of copper compounds and their main properties are presented as well the importance of this element in biological systems and organic reactions is explored, showing how essential copper is for life and society.
\end{abstract}

Keywords: tabela periódica; mineração; ciclo redox; cobre em síntese.

\section{INTRODUÇÃO}

A Assembleia Geral das Nações Unidas proclamou o ano de 2019 como sendo o Ano Internacional da Tabela Periódica dos Elementos Químicos (International Year of the Periodic Table of Chemical Elements - IYPT 2019). ${ }^{1}$ Este fato está associado aos 150 anos do estabelecimento da Tabela Periódica dos Elementos Químicos uma vez que, em 1869, o cientista russo Dmitri Mendeleev publicou sua contribuição ao sistema periódico dos elementos químicos, cujo título (numa tradução livre) é "Sobre as relações de propriedades com os pesos atômicos dos elementos". Não deixa de ser surpreendente que um artigo revolucionário como este ocupe o espaço correspondente a apenas uma página da revista científica Zeitschrift für Chimie! ? $^{2}$

A Tabela Periódica é uma das entidades mais populares da Química pois é facilmente reconhecida por parte da população escolarizada, passando a ser a representação dessa ciência.

Não tão popular assim é a compreenção de que muitos elementos da Tabela Periódica são produzidos aqui no Brasil e representam uma importante fonte de divisas para a nação. O cobre é um deles, e é conhecido e utilizado pela civilização há mais de seis mil anos, especialmente em sua forma metálica e como componente de ligas especiais. Em Química, há fatores que fazem do cobre um elemento importante. Um primeiro e fundamental fator é que sais de cobre possuem baixa toxicidade e são relativamente baratos, facilitando o acesso e o uso.

\section{ASPECTOS GERAIS DA QUÍMICA DO ELEMENTO CO- BRE}

Último metal de transição da primeira série do bloco $d$, o cobre ( $Z=29$ ) está localizado no grupo 11, conhecido como o grupo dos metais de cunhagem $(\mathrm{Cu}, \mathrm{Ag}, \mathrm{Au})$. Embora apresente configuração eletrônica $[A r] 3 d^{10} 4 s^{I}$, sua química é dominada pelo $\mathrm{Cu}(\mathrm{II})$, cuja configuração eletrônica é $[A r] 3 d^{9}$, com subnível $(n-1) d$ incompleto e, portanto, um elemento de transição segundo definição da IUPAC. O Cu(II), sistema $d^{9}$, coordena-se a 4,5 ou 6 ligantes em geometrias quadrática, piramidal ou octaédrica com distorção de Jahan-Teller, enquanto que o $\mathrm{Cu}(\mathrm{I})$, sistema $d^{10}$, apresenta números

*e-mail: silviodc@ufba.br

\#e-mail alternativo: las@ufba.br de coordenação mais baixos, 3 e 4, e geometrias tetraédrica ou trigonal plana, Figura 1.

Devido ao caráter de ácido de Lewis de fronteira, o $\mathrm{Cu}$ (II) tem afinidade por bases de Lewis duras como íons $\mathrm{O}^{2-}, \mathrm{OH}^{-}$e $\mathrm{CO}_{3}^{2-}$ e por bases de Lewis macias como o íon $\mathrm{S}^{2-}$. Já o $\mathrm{Cu}(\mathrm{I})$ apresenta caráter de ácido de Lewis macio e tem maior afinidade por bases também macias. Por essa razão, o cobre ocorre na natureza, majoritariamente, na forma de minérios sulfetados, especialmente nos depósitos subterrâneos; mas também é encontrado como óxidos, carbonatos, hidroxissilicatos e sulfatos nas camadas superiores dos depósitos minerais, onde o ambiente é mais oxidante..$^{3,4}$

\section{Complexos de cobre(l)}

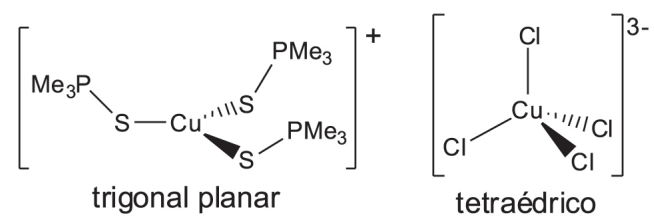

\section{Complexos de cobre(II)}

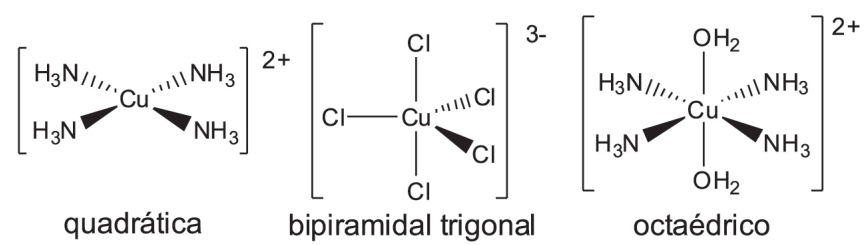

Figura 1. Complexos de $\mathrm{Cu}(\mathrm{I})$ e $\mathrm{Cu}(\mathrm{II})$ em diferentes ambientes químicos, números de coordenação e geometrias

\section{PRODUÇÃO INDUSTRIAL DO COBRE}

As minas de produção de minério de cobre no Brasil ocorrem apenas nos Estados do Pará, Goiás e Bahia, segundo a edição mais recente do Anuário Mineral Brasileiro (edição de 2018, referentes aos dados consolidados do ano de 2017) publicado pela Agência Nacional de Mineração. ${ }^{5}$ Essa é também a ordem da produção bruta de minério pelos Estados citados que totaliza, para o ano em questão, 111.340.233 toneladas de minério bruto assim distribuídos na 
produção nacional: Pará 68,8\%, Goiás 30,0\% e Bahia 1,2\%. Apenas cerca de $1 \%$ da produção bruta é beneficiada (1.283.760 t), que é a produção anual das usinas de tratamento.

Quando se analisa o volume de minério que é benficiado (1,2 milhões de toneladas), a produção nacional anual é distribuída entre Pará $77 \%$, Goiás $19 \%$ e Bahia 4\%. Todavia, ao se relacionar a produção beneficiada de cada Estado em relação à produção bruta local, o cenário muda para Bahia beneficiando 3,2\% do minério de cobre que produz, Pará $1,3 \%$ e Goiás $0,7 \%$. Como a produção comercializada é de apenas 1.279 .093 toneladas, o que representa $1,1 \%$ de toda produção bruta nacional, claro fica que ainda há um longo caminho de industrialização a percorrer.

Apesar desse cenário de baixo beneficamento do minério, em 2017 o cobre ocupou o segundo lugar na participação $(9,4 \%)$ entre as principais substâncias metálicas no valor da produção mineral comercializada, perdendo apenas para o ferro $(71,1 \%)$, seguido de ouro $(8,9 \%)$, alumínio $(3,7 \%)$ e níquel $(2,5 \%) .^{5}$

A Tabela 1 apresenta os principais minerais de cobre com suas estruturas e os respectivos teores máximos desse metal.

A abundância relativa de cobre na crosta terrestre é de 68 ppm, e estima-se que cerca de $50 \%$ de todos os depósitos do metal estejam na forma de calcopirita $\left(\mathrm{CuFeS}_{2}\right),{ }^{6}$ um mineral sulfetado com $35 \%$ de cobre associado ao ferro (Tabela 1). O cobre é largamente empregado na indústria de geração e transmissão de energia elétrica devido à associação de duas propriedades marcantes, elevada condutividade elétrica $\left(61,7 \mathrm{~S} \mathrm{~m} \mathrm{~mm}^{-2}\right)$ e resistência à corrosão $\left(\mathrm{E}_{\mathrm{r}}{ }^{\mathrm{o}}=+0,34 \mathrm{~V}\right), \mathrm{o}$ que justifica a extensa extração e beneficiamento de seus minérios. Cerca de $80 \%$ da produção primária de cobre provem de minérios sulfetados, porém de baixa qualidade, com cerca de $0,5-2,0 \%$ do metal, exigindo uma etapa prévia de enriquecimento para obter o concentrado com cerca de $20-30 \%$ de cobre. ${ }^{6,7}$ Nesse caso, o processo empregado de extração e refino é o pirometalúrgico. Os $20 \%$ restantes da produção primária de cobre são provenientes de minerais oxidados de baixo teor de cobre e, nesse caso, o processo mais apropriado para a extração é o hidrometalúrgico. ${ }^{7}$

A etapa de enriquecimento do minério sulfetado, denominada flotação a espuma (froth-flotation), ${ }^{4}$ é precedida pelos processos de britagem e moagem do minério irregular, os quais geram um passivo ambiental com acúmulo de grande quantidade de um fino pó como resíduo. Após a moagem, é adicionada ao minério finamente dividido uma mistura contendo água, um reagente coletor, espécies químicas com caráter polar e apolar, e detergente que tem como finalidade diminuir a tensão superficial na interface líquido/ar. Nessa etapa, o reagente coletor, geralmente um xantato, adsorve-se à superfície do mineral sulfetado contido no pó finamente dividido, interagindo pela parte polar da estrutura e deixando a parte apolar exposta. $\mathrm{O}$ caráter hidrofóbico conferido às partículas de sulfeto revestidas com o reagente coletor permite a adesão bolha-partícula com a injeção de ar à mistura. Com a agitação da polpa, as bolhas são arrastadas até a superfície, onde são removidas como uma espuma contendo o concentrado fino. Após lavagem e secagem, o concentrado de cobre é, então, tratado por métodos pirometalúrgicos de extração e refino, e em seguida submetido à eletrólise para obter cobre de alta pureza. A Figura 2 ilustra todas as etapas envolvidas na transformação do cobre mineral em metal por extração pirometalúrgica e refino eletrolítico.

As principais etapas da extração pirometalúrgica incluem a fundição do concentrado de cobre a $1400{ }^{\circ} \mathrm{C}$ em presença de ar, seguida da adição de sílica. ${ }^{6}$ Nessa condição, o FeS é mais facilmente convertido ao óxido que $\mathrm{Cu}_{2} \mathrm{~S}$.

$2 \mathrm{CuFeS}_{2}(\mathrm{l})+4 \mathrm{O}_{2}(\mathrm{~g}) \stackrel{1400{ }^{\circ} \mathrm{C}}{\longrightarrow} 2 \mathrm{FeO}(\mathrm{l})+\mathrm{Cu}_{2} \mathrm{~S}(\mathrm{l})+3 \mathrm{SO}_{2}(\mathrm{~g})$

A adição de sílica e ar auxilia na conversão do FeS remanescente em $\mathrm{FeO}$ e posteriormente em silicato de ferro. Nessa etapa, o silicato de ferro líquido é separado da escória, considerada um grande passivo ambiental que se acumula em montanhas na área externa da fábrica. Já $\mathrm{Cu}_{2} \mathrm{~S}$ é parcialmente convertido a $\mathrm{Cu}_{2} \mathrm{O}$ e, então, a cobre metálico. As equações 2-5 representam as principais reações químicas que ocorrem na etapa de conversão.

$$
\begin{gathered}
2 \mathrm{FeS}(\mathrm{l})+3 \mathrm{O}_{2}(\mathrm{~g}) \rightarrow 2 \mathrm{FeO}(\mathrm{l})+2 \mathrm{SO}_{2}(\mathrm{~g}) \\
\mathrm{FeO}(\mathrm{l})+\mathrm{SiO}_{2}(\mathrm{l}) \rightarrow \mathrm{FeSiO}_{3}(\mathrm{l}) \\
2 \mathrm{Cu}_{2} \mathrm{~S}(\mathrm{l})+3 \mathrm{O}_{2}(\mathrm{~g}) \rightarrow 2 \mathrm{Cu}_{2} \mathrm{O}(\mathrm{l})+2 \mathrm{SO}_{2}(\mathrm{~g}) \\
2 \mathrm{Cu}_{2} \mathrm{O}(\mathrm{l})+\mathrm{Cu}_{2} \mathrm{~S}(\mathrm{l}) \rightarrow 6 \mathrm{Cu}(\mathrm{l})+\mathrm{SO}_{2}(\mathrm{~g})
\end{gathered}
$$

Após remoção do ferro na forma de silicato, obtém-se o mate com $45-60 \%$ de cobre e a etapa de conversão dá origem ao cobre blister com $98,5 \%$ de pureza. A etapa posterior, de refino a fogo, resulta no cobre anódico com 99,7\% de pureza, que segue para o refino eletrolítico para obtenção do cobre catódico com pureza superior a 99,99\%.6.7

O processo de refino eletrolítico elimina praticamente todas as impurezas do cobre anódico obtido do refino a fogo, especialmente, oxigênio, enxofre e alguns metais remanescentes que diminuem sua condutividade elétrica. ${ }^{6,7}$ Nesse processo, as placas de cobre anódico são imersas em uma solução ácida de sulfato de cobre nas quais também são imersas as chapas catódicas, que podem ser finas placas de cobre puro que são incorporadas ao cátodo durante a eletrólise ou outro metal passivado resistente à corrosão, como titânio, que servem de suporte para deposição do cobre catódico. Resumindo, ao aplicar a descarga elétrica, o cobre com 99,7\% de pureza é oxidado no ânodo e ao mesmo tempo sofre deposição no cátodo com pureza superior a 99,99\% (equações 6 e 7). O resíduo sólido da oxidação do cobre anódico depositado no fundo das cubas eletrolíticas, conhecido como lama anódica, é fonte de metais preciosos como ouro e prata.

$$
\begin{aligned}
& \text { Ânodo (+): } \mathrm{Cu}(\mathrm{s}) \rightarrow \mathrm{Cu}^{2+}(\mathrm{aq})+2 \mathrm{e} \\
& \quad \text { (resíduo sólido: lama anódica })
\end{aligned}
$$

$$
\text { Cátodo (-): } \mathrm{Cu}^{2+}(\mathrm{aq})+2 \mathrm{e}^{-} \rightarrow \mathrm{Cu}(\mathrm{s})
$$

Tabela 1. Principais minerais de cobre, estruturas e teores máximos do metal ${ }^{3,4}$

\begin{tabular}{ccccc}
\hline Tipo & Mineral & Fórmula & \% Teórico de Cu & Estrutura \\
\hline Sulfeto & Calcopirita & $\mathrm{CuFeS}_{2}$ & 35 & Tetragonal \\
& Bornita & $\mathrm{Cu}_{5} \mathrm{FeS}_{4}$ & 63 & Ortorrômbica \\
& Calcocita & $\mathrm{Cu}_{2} \mathrm{~S}$ & 79,8 & Ortorrômbica, Hexagonal \\
\hline \multirow{2}{*}{ Óxido } & Cuprita & $\mathrm{Cu}_{2} \mathrm{O}$ & 88,8 & Cúbica de corpo centrado \\
& Tenorita & $\mathrm{CuO}$ & 79,9 & Monoclínica \\
\hline \multirow{2}{*}{ Carbonato } & Malaquita & $\mathrm{Cu}_{2} \mathrm{CO}_{3}(\mathrm{OH})_{2}$ & 58 & Monoclínica \\
& Azurita & $\mathrm{Cu}_{3}\left(\mathrm{CO}_{3}\right)_{2}(\mathrm{OH})_{2}$ & 55 & Monoclínica \\
\hline
\end{tabular}




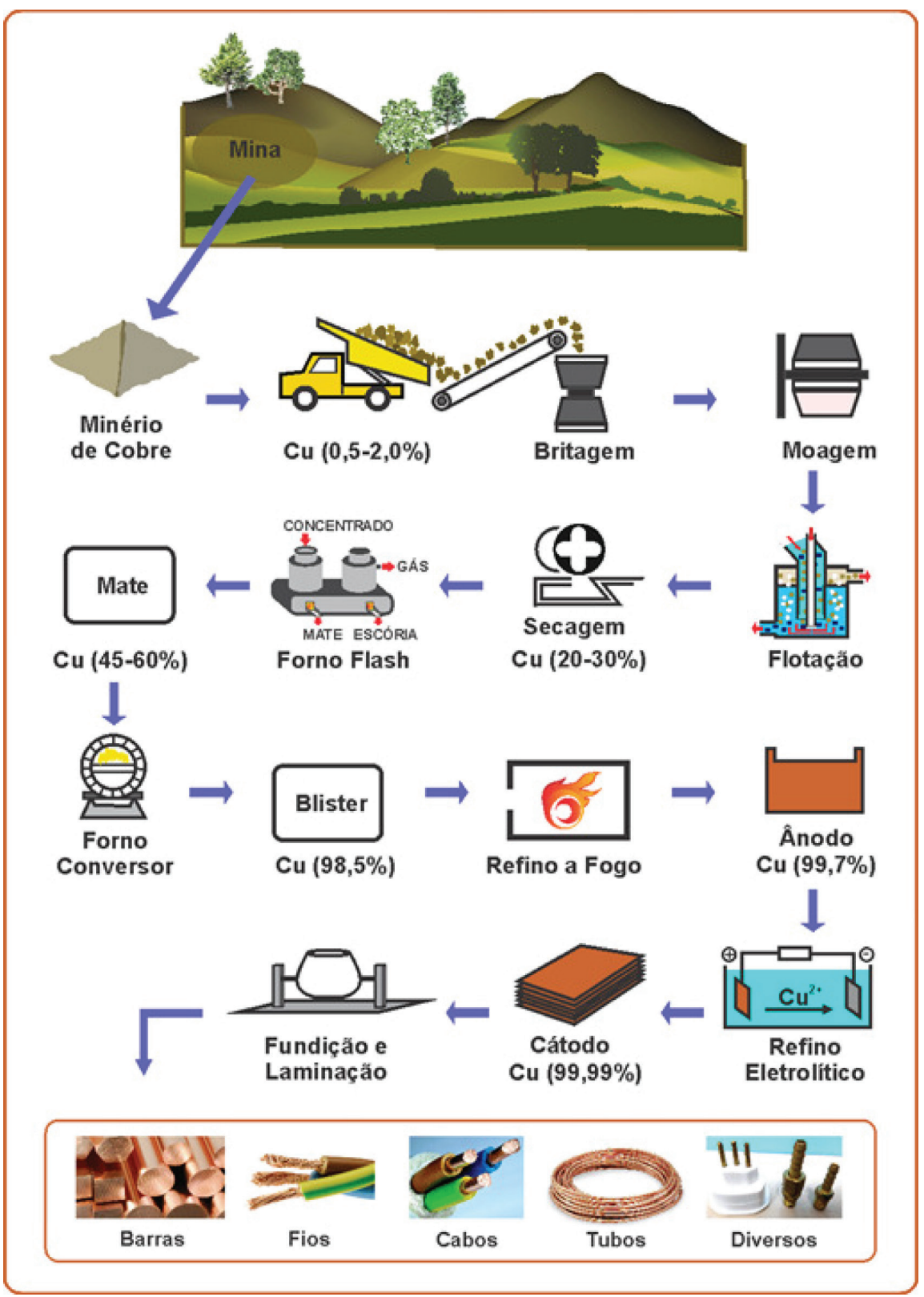

Figura 2. Etapas do beneficiamento do minério de cobre sulfetado da mina até o produto final

A companhia Paranapanema S.A. é a única empresa no Brasil que transforma cobre mineral em metal. A matriz localizada em Dias d'Ávila - BA, a Caraíba Metais S.A., atua na fundição e no refino de cobre primário. Lá são produzidos cátodos, fios e vergalhões, além de coprodutos de cobre resultantes dos processos pirometalúrgicos do minério sulfetado e refino eletrolítico, como ácido sulfúrico, pelo aproveitamento do dióxido de enxofre produzido durante as etapas de extração, silicato de ferro, resultante da etapa de conversão, e lama anódica, resultante da etapa de refino eletrolítico de onde são extraídos metais preciosos. ${ }^{8}$

\section{CORRELAÇÕES ENTRE A QUÍMICA DO COBRE E SUAS APLICAÇÕES}

No estado sólido, o cobre metálico adquire estrutura cristalina cúbica de face centrada, ou empacotamento cúbico denso, que, em função do arranjo em camadas empilhadas, um plano pode se deslocar sobre outro, o que torna o metal muito maleável e dúctil. Assim, o cobre catódico, que consiste em placas regulares de alta pureza, é empregado como matéria-prima para produção de vergalhões e barramentos. Os vergalhões são empregados na produção de cabos condutores para transmissão de energia elétrica e térmica, eletroeletrônicos, barras coletoras, contatos elétricos, telefonia, telecomunicações, transmissão de energia, indústria automobilística, construção civil e também no setor de informática. ${ }^{8} \mathrm{O}$ cobre é ainda empregado na composição de diversas ligas, como o bronze, liga de cobre e estanho (7 - 10\% de Sn) e o latão, liga de cobre e zinco de composição variada, podendo resultar nas fases $\alpha, \beta$ e $\gamma$, a depender da razão $\mathrm{Cu} / \mathrm{Zn} .^{6}$

Embora apresente caráter nobre como consequência da alta entalpia de sublimação e das elevadas energias de ionização, o cobre participa de reações de oxirredução catalíticas em diversos processos, inclusive biológicos. Semelhante ao que ocorre com outros metais, o cobre é um elemento essencial para os organismos vivos e sua química redox está envolvida em uma variedade de processos de oxidação. Tanto $\mathrm{Cu}(\mathrm{I})$ quanto $\mathrm{Cu}$ (II) tem afinidade por bases macias, sítios, em geral, presentes em ligantes biológicos, o que permite desempenhar várias funções celulares, tais como processos metabólicos e funções em órgãos humanos. 
O cobre é o terceiro elemento do bloco $d$ mais abundante no corpo humano, ficando atrás apenas de ferro e zinco. A quantidade desse elemento em um indivíduo adulto é de cerca de $100 \mathrm{mg}$ e requer um consumo diário de 3-5 mg. ${ }^{6} \mathrm{~A}$ absorção de cobre na dieta (alimento, suplementos e água) ocorre principalmente na forma de $\mathrm{Cu}(\mathrm{II})$, mas parte é reduzida a $\mathrm{Cu}(\mathrm{I})$ por enzimas redutases. $\mathrm{O}$ corpo humano possui um sofisticado maquinário que disponibiliza o cobre quando esse é necessário e, ao mesmo tempo, o elimina quando está em excesso. Essa regulação, conhecida como homeostase de cobre, ${ }^{9}$ depende de uma rede de proteínas e outras moléculas biológicas que se encarregam da absorção, transporte, distribuição, armazenamento e excreção de cobre. As denominadas proteínas azuis de cobre, que apresentam intensa absorção em torno de $600 \mathrm{~nm}$, catalisam reações redox em organismos que vão desde bactérias a seres humanos devido ao elevado potencial de redução, que pode variar de $0,2 \mathrm{~V}$ a $1,0 \mathrm{~V}$ a depender da proteína à qual o cobre está ligado, bem como condições de $\mathrm{pH} .{ }^{10}$ Esses valores são considerados anômalos em comparação aos complexos inorgânicos de cobre em solução aquosa. O elevado potencial de redução é atribuído ao impedimento estérico nos sítios de cobre, $\mathrm{Cu}(\mathrm{II})-\mathrm{L}$ e $\mathrm{Cu}(\mathrm{I})-\mathrm{L} \cdot$, em geometria trigonal planar $(\mathrm{L}=$ dois ligantes $\mathrm{N}$-imidazólicos e um S-tiolato de proteínas azuis, tais como azurina e plastocianina) e a coloração azul é consequência de transição de transferência de carga cobre-ligante. ${ }^{10}$ Essas propriedades explicam a boa capacidade de transferência de carga na mediação de reações redox. Por outro lado, em função das propriedades redox e capacidade de gerar radicais livres, o cobre exerce efeitos deletérios em doenças neurodegenerativas, uma vez que é capaz de se ligar às proteínas envolvidas na neurodegeneração, promovendo a sua desnaturação. ${ }^{11}$

Em solução aquosa, $\mathrm{Cu}(\mathrm{I})$ desproporciona em $\mathrm{Cu}^{0}$ e $\mathrm{Cu}(\mathrm{II})$, mas no estado sólido há possibilidade de se estabilizar as diferentes espécies de cobre, formando compostos heterovalentes. $\mathrm{O}$ sal de Chevreul é um exemplo disso, capaz de estabilizar $\mathrm{Cu}(\mathrm{I})$ e $\mathrm{Cu}(\mathrm{II})$ em uma rede de sulfito na qual o $\mathrm{Cu}(\mathrm{I})$ coordena-se tanto através de átomos de enxofre quanto através de átomos de oxigênio em ambiente tetraédrico; enquanto $\mathrm{Cu}$ (II) coordena-se ao íon sulfito apenas através de átomos de oxigênio em ambiente octaédrico. ${ }^{12}$

$\mathrm{O}$ sal de Chevreul, $\mathrm{Cu}_{2} \mathrm{SO}_{3} \cdot \mathrm{CuSO}_{3} \cdot 2 \mathrm{H}_{2} \mathrm{O}$, com coloração vermelho-tijolo intensa, foi preparado pela primeira vez em 1812 por Michel Chevreul, ${ }^{13}$ que se dedicava aos estudos de gorduras de origem animal, mas também debruçou-se sobre o entendimento da origem das cores nos compostos. Porém, a origem da coloração intensa do sal de Chevreul só foi elucidada quase dois séculos depois com a interpretação dos espectros eletrônicos por meio de cálculos de modelagem molecular, tomando como referência o dímero $\left[\mathrm{Cu}_{2}^{\mathrm{I}}\left(\mathrm{SO}_{3}\right)_{2}\left(\mathrm{SO}_{3}\right)_{2}\right]^{6-}$ ligado a dois fragmentos $\left[\mathrm{Cu}^{\mathrm{II}}\left(\mathrm{H}_{2} \mathrm{O}\right)_{2}\left(\mathrm{SO}_{3}\right)_{2}\right]^{2-}$ para simular grupos repetitivos $\left\{\mathrm{Cu}_{2}^{\mathrm{I}}\left(\mathrm{SO}_{3}\right)_{2}\left[\mathrm{Cu}^{\mathrm{II}}\left(\mathrm{H}_{2} \mathrm{O}\right)_{2}\left(\mathrm{SO}_{3}\right)_{2}\right]_{2}\right\}^{6-}$ da estrutura cristalina, representado na Figura $3 .{ }^{14} \mathrm{O}$ espectro eletrônico do sal de Chevreul (Figura 3) consiste de uma banda de transferência de intervalência $\mathrm{Cu}^{\mathrm{I}}-\mathrm{Cu}^{\mathrm{II}}$ em $500 \mathrm{~nm}$, responsável pela sua coloração vermelha característica. Além disso, apresenta uma banda de transferência de carga em $425 \mathrm{~nm}$ associada ao cromóforo $\left[\mathrm{Cu}_{2}^{\mathrm{I}}\left(\mathrm{SO}_{3}\right)_{2}\left(\mathrm{SO}_{3}\right)_{2}\right]^{6-}$, e duas bandas de transições de campo ligante a 785 e 1000 nm envolvendo distorção de Jahn-Teller dos níveis do Cu(II). A banda de intervalência desaparece quando $\mathrm{Cu}$ (II) é substituído por outros cátions $\mathrm{M}(\mathrm{II})$, como $\mathrm{Mn}(\mathrm{II}), \mathrm{Fe}(\mathrm{II})$ e Cd(II), porém a banda de transferência de carga sobre o cromóforo contendo $\mathrm{Cu}(\mathrm{I})$ é preservada, com algum deslocamento, resultando em compostos isomórficos com colorações distintas, Figura 3.

Os materiais à base de cobre são capazes de promover uma variedade de reações, principalmente devido a seus estados de oxidação acessíveis $(\mathrm{Cu}(0), \mathrm{Cu}(\mathrm{I})$ e $\mathrm{Cu}(\mathrm{II}))$. Os óxidos de cobre são materiais promissores para aplicações em transformações catalíticas de substâncias orgânicas, eletrotrocatálise e fotocatálise, principalmente

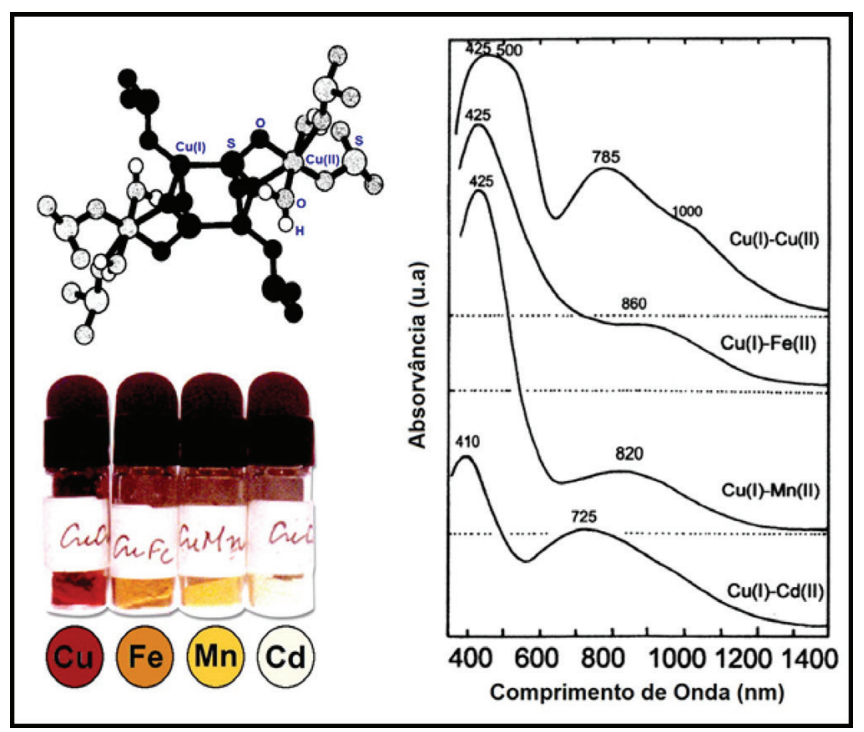

Figura 3. Sulfitos duplos de valência mista do tipo $\mathrm{Cu}_{2} \mathrm{SO}_{3} \cdot \mathrm{MSO}_{3} \cdot 2 \mathrm{H}_{2} \mathrm{O}$ $(M=C u, F e, M n$ e $C d)$ e respectivos espectros eletrônicos. A estrutura representa o grupo $\left\{\mathrm{Cu}_{2}^{\mathrm{I}}\left(\mathrm{SO}_{3}\right)_{2}\left[\mathrm{Cu}^{\mathrm{II}}\left(\mathrm{H}_{2} \mathrm{O}\right)_{2}\left(\mathrm{SO}_{3}\right)_{2}\right]_{2}\right\}^{6}$, mostrando a unidade $\left[\mathrm{Cu}_{2}^{\mathrm{I}}\left(\mathrm{SO}_{3}\right)_{2}\left(\mathrm{SO}_{3}\right)_{2}\right]^{6-}$ (cromóforo, parte escura) ao centro e os fragmentos $\left[\mathrm{Cu}^{\mathrm{II}}\left(\mathrm{H}_{2} \mathrm{O}\right)_{2}\left(\mathrm{SO}_{3}\right)_{2}\right]^{2-}$ (parte clara). Adaptado da ref. 14

devido ao seu alto potencial redox, baixa toxicidade e baixo custo. ${ }^{15}$ Catalisadores a base de cobre são muito empregados na hidrogenação seletiva da ligação C-O presente em ésteres, ácidos carboxílicos, éteres, furfural e $\mathrm{CO}_{2} \cdot{ }^{15}$ No entanto, não há um consenso acerca do mecanismo de reação, especialmente com relação ao papel desempenhado pelas espécies de cobre. Uma das hipóteses sugere que apenas $\mathrm{Cu}^{0}$ ou $\mathrm{Cu}(\mathrm{I})$ são os sítios ativos, outra sugere que ocorre uma contribuição sinérgica entre as duas espécies de cobre. A proposta de que $\mathrm{Cu}^{0}$ e $\mathrm{Cu}(\mathrm{I})$ estão envolvidos em um ciclo dinâmico durante o processo de hidrogenação é mais consensual, no qual espécies de $\mathrm{Cu}(\mathrm{I})$ na superfície atuam como sítios ácidos de Lewis que facilitam a adsorção e ativação da ligação $\mathrm{C}-\mathrm{O}$, enquanto os sítios $\mathrm{Cu}^{0}$ atuam adsorvendo hidrogênio molecular. ${ }^{16,17}$ Os óxidos de cobre são largamente empregados como catalisadores em reações de remediação ambiental, incluindo redução catalítica de $\mathrm{NO}_{x}$ em amônia, combustão catalítica de compostos orgânicos voláteis, oxidação de $\mathrm{CO}$, degradação de poluentes orgânicos e oxidação catalítica de fuligem. ${ }^{15}$

Espécies de cobre $\left(\mathrm{Cu}^{0}, \mathrm{Cu}(\mathrm{I})\right.$ e $\left.\mathrm{Cu}(\mathrm{II})\right)$ associadas ao dióxido de titânio são as mais empregadas como cocatalisadores na conversão fotocatalítica de $\mathrm{CO}_{2}$ em combustíveis solares como $\mathrm{CO}, \mathrm{CH}_{4}$, $\mathrm{CH}_{3} \mathrm{OH}, \mathrm{HCHO}, \mathrm{HCOOH}$ e $\mathrm{C}_{2} \mathrm{H}_{5} \mathrm{OH} \cdot{ }^{18-23}$ As espécies de cobre no fotocatalisador podem estar presentes de diferentes formas: como dopante por substituição de alguns $\mathrm{Ti}(\mathrm{IV})$ por $\mathrm{Cu}$ (II) ou por $\mathrm{Cu}$ (I), ou na forma de heteroestruturas de $\mathrm{TiO}_{2} \operatorname{com~} \mathrm{Cu}_{2} \mathrm{O}, \mathrm{CuO}$ ou $\mathrm{Cu}^{0}$. Do ponto de vista termodinâmico, a capacidade do cobre dopante atuar como um aprisionador de elétrons está associada ao potencial de redução dos íons metálicos serem mais positivos $\left(\mathrm{E}^{\circ}\left(\mathrm{Cu}^{+} / \mathrm{Cu}\right)=+0,52 \mathrm{~V}\right.$ e $\left.\mathrm{E}^{\circ}\left(\mathrm{Cu}^{2+} / \mathrm{Cu}\right)=+0,34 \mathrm{~V}\right)$ que o potencial da borda da banda de condução do $\mathrm{TiO}_{2}(-0,2 \mathrm{~V})$, criando um gradiente de potencial que favorece o processo de transferência de elétrons (Figura 4). ${ }^{19}$ Sendo assim, esperava-se que o $\mathrm{Cu}(\mathrm{I})$ atuasse como um aprisionador de elétrons mais eficiente que o $\mathrm{Cu}(\mathrm{II})$, evitando processos de recombinação de cargas no semicondutor fotoexcitado. Entretanto, exatamente pela forte interação entre $\mathrm{TiO}_{2}$ e $\mathrm{Cu}(\mathrm{I})$ introduzido nas vacâncias, resulta no aprisionamento profundo do elétron transferido da borda da banda de condução do $\mathrm{TiO}_{2}$ fotoexcitado. Como consequência, os elétrons terão mais dificuldade em serem transferidos para as espécies adsorvidas à superfície. 


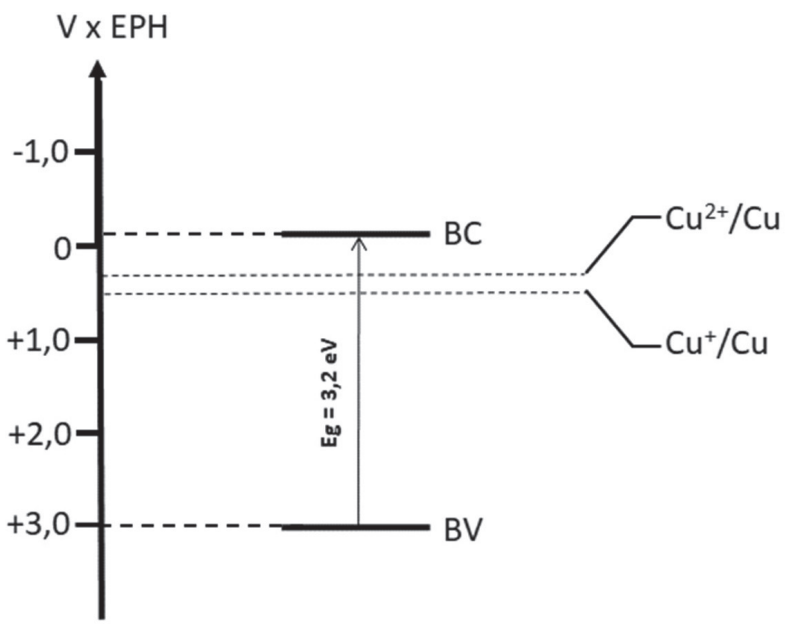

Figura 4. Diagrama de energia de $\mathrm{TiO}_{2}$ com o posicionamento das bordas das bandas de condução e valência e respectivos potenciais padrão dos pares $\mathrm{Cu}^{+} / \mathrm{Cu}$ e $\mathrm{Cu}^{2+} / \mathrm{Cu}$ contra o Eletrodo Padrão de Hidrogênio (EPH)

A energia de bandgap do $\mathrm{TiO}_{2}$ é $3,2 \mathrm{eV}$, enquanto que as energias de bandgap de $\mathrm{CuO}$ e $\mathrm{Cu}_{2} \mathrm{O}$ são $1,41 \mathrm{eV}$ e 2,17 eV, respectivamente. Na formação da heterojunção entre os dois semicondutores, o gradiente de potencial gerado é maior na heteroestrutura $\mathrm{CuO} / \mathrm{TiO}_{2}$ comparada à heteroestrutura $\mathrm{Cu}_{2} \mathrm{O} / \mathrm{TiO}_{2}$, em função do posicionamento dos potenciais das bordas de valência e condução, como ilustrado no esquema da Figura 5 ( $\mathrm{a} \mathrm{e} \mathrm{b).} \mathrm{Atribui-se} \mathrm{a} \mathrm{isso} \mathrm{o} \mathrm{fato} \mathrm{da} \mathrm{atividade}$ fotocatalítica na fotorredução de $\mathrm{CO}_{2}$ da heteroestrutura $\mathrm{CuO} / \mathrm{TiO}_{2}$ ser superior a de $\mathrm{Cu}_{2} \mathrm{O} / \mathrm{TiO}_{2}{ }^{18-23}$ Já no caso de nanopartículas de cobre elementar depositadas sobre o semicondutor, o metal desempenha papel semelhante à platina na maioria dos sistemas fotocatalíticos, em que os elétrons fotogerados com a iluminação do semicondutor migram para as nanopartículas do metal, através da heterojunção, Figura 5 (c). A barreira de Schottky na interface semicondutor/metal impede que os elétrons se recombinem com as lacunas fotogeradas no semicondutor. ${ }^{23,24} \mathrm{O}$ aprisionamento raso de elétrons permite sua transferência para espécies adsorvidas à superfície.

O armazenamento de energia no ciclo redox de óxidos metálicos é considerado uma tecnologia promissora de armazenamento de energia termoquímica. ${ }^{25,26} \mathrm{O}$ sistema opera a altas temperaturas, usando ar como reagente e meio de transferência de calor, e a separação dos produtos ocorre de forma simples, visto que encontram-se em fases distintas. As reações típicas no processo de armazenamento de energia são representadas pelas equações 8 e $9 .{ }^{25}$

$$
\begin{aligned}
& \text { Redução: } \mathrm{Me}_{\mathrm{x}} \mathrm{O}_{\mathrm{y}} \rightarrow \mathrm{Me}_{\mathrm{x}} \mathrm{O}_{\mathrm{y}-1}+1 / 2 \mathrm{O}_{2} \quad \Delta \mathrm{H}>0 \\
& \text { Oxidação: } \mathrm{Me}_{\mathrm{x}} \mathrm{O}_{\mathrm{y}-1}+1 / 2 \mathrm{O}_{2} \rightarrow \mathrm{Me}_{\mathrm{x}} \mathrm{O}_{\mathrm{y}} \quad \Delta \mathrm{H}<0
\end{aligned}
$$

A reação de redução ocorre com fornecimento de energia e, em geral, requer temperaturas muito elevadas. Já a reação de oxidação ocorre com a liberação da energia armazenada. Vários pares de óxidos já foram investigados para essa aplicação, tais como $\mathrm{BaO}_{2} / \mathrm{BaO}, \mathrm{Co}_{3} \mathrm{O}_{4} / \mathrm{CoO}$, $\mathrm{Mn}_{2} \mathrm{O}_{3} / \mathrm{Mn}_{3} \mathrm{O}_{4}, \mathrm{Fe}_{2} \mathrm{O}_{3} / \mathrm{Fe}_{3} \mathrm{O}_{4}$ e $\mathrm{CuO} / \mathrm{Cu}_{2} \mathrm{O}$. O par $\mathrm{CuO} / \mathrm{Cu}_{2} \mathrm{O}$ requer temperatura de redução elevada, de $1030^{\circ} \mathrm{C}$, mas tem alta densidade de armazenamento de energia ( $\left.811 \mathrm{~kJ} \mathrm{~kg}^{-1}\right)$, muito superior ao $\mathrm{BaO}_{2} / \mathrm{BaO}$ (432 $\mathrm{kJ} \mathrm{kg}^{-1}$ ), um dos primeiros pares estudados. ${ }^{25}$ Durante a carga, o sistema utiliza uma fonte externa de calor para reduzir e fundir $\mathrm{CuO}$ sólido, resultando na mistura líquida $\mathrm{CuO} / \mathrm{Cu}_{2} \mathrm{O}$. A fonte de calor pode ser por aquecimento Joule usando o excedente de eletricidade da rede elétrica ou energia renovável (solar/eólica). Dessa forma, a energia fornecida nesse processo de redução e fusão é armazenada nas formas de calor sensível e latente e energia termoquímica. Durante a descarga, a mistura $\mathrm{CuO} / \mathrm{Cu}_{2} \mathrm{O}$ fundida é oxidada e resfriada em um reator de oxidação usando ar. Nessa etapa, a energia armazenada é liberada e transferida para o ar submetido à alta pressão e alta temperatura, e esta pode ser convertida em diferentes formas.

O cobre é o material mais amplamente empregado na indústria de confecção de destiladores para produção de cachaça em alambiques. Durante o processo de destilação, a superfície quente do destilador em contato com o fermentado rico em compostos voláteis faz com que o cobre atue como catalisador em várias reações, tais como esterificação, desidratação e oxidação. ${ }^{27} \mathrm{~A}$ presença de cobre no destilador também está associada aos baixos níveis de compostos sulfurados na bebida, como dimetilsulfeto (DMS), e toxicidade dos aldeídos e íons cobre(II). ${ }^{28}$ Vários materiais alternativos para construção de destiladores já foram propostos no sentido de controlar a contaminação do destilado por íons $\mathrm{Cu}$ (II), para atender exigências do mercado internacional e aumentar o volume de exportação do produto. Dentre elas estão o aço inox, alumínio e porcelana; mas a ausência de cobre no processo produz alterações sensoriais na bebida (cor, sabor, aroma), apresentando aroma típico de sulfeto. Dentre os materiais já estudados, o que mais se assemelha ao cobre é o aço inox, porém apresenta a desvantagem de produzir um destilado final com teores elevados de DMS. ${ }^{29}$

Há ainda outras aplicações do cobre metálico de alta pureza em menor escala, como, por exemplo, os dispositivos intrauterinos (DIU) que, em geral, são constituídos de polietileno recoberto com cobre nas hastes vertical e horizontal. No mecanismo de ação do DIU, admite-se que uma pequena quantidade de íons cobre bloqueia os

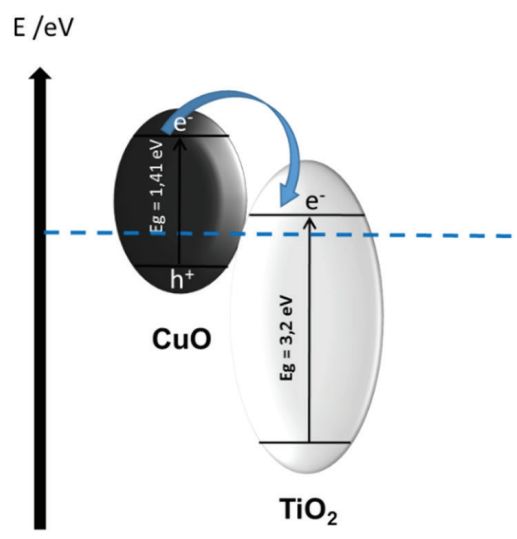

(a) Heterojunção $\mathrm{CuO} / \mathrm{TiO}_{2}$

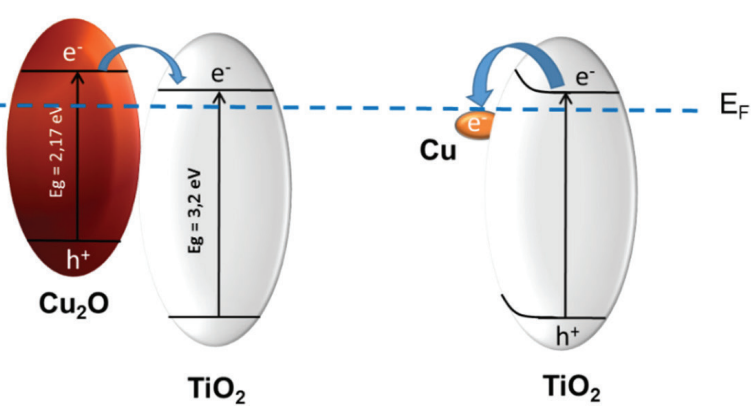

(b) Heterojunção $\mathrm{Cu}_{2} \mathrm{O} / \mathrm{TiO}_{2}$ (c) Heterojunção $\mathrm{Cu} / \mathrm{TiO}_{2}$

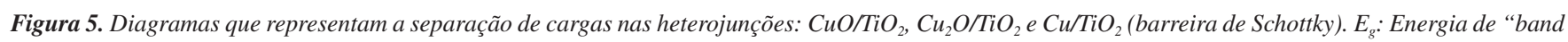
gap"; $E_{F}$ : Energia do nível de Fermi; $h^{+}$: lacuna fotogerada; $e^{-}$: elétron fotogerado 
espermatócitos, inibindo a migração de espermatozóides transtubais, o que previne a formação de zigotos. ${ }^{30}$

\section{PRINCIPAIS REAÇÕES ORGÂNICAS MEDIADAS POR COBRE}

Várias reações orgânicas são catalisadas ou promovidas por cobre e seus derivados, com ampla aplicação na pesquisa e na indústria. Dependendo de seu estado de oxidação, o cobre pode intervir e participar de reações que envolvam 1 ou 2 elétrons, através de mecanismos radicalares e/ou iônicos. ${ }^{31}$ Cobre também se coordena com facilidade a hetero-átomos e ligações pi, e é conhecido ativante de alcinos terminais. Reações clássicas como acoplamentos cruzados C-C de Ullmann, ${ }^{32}$ Castro-Stephens ${ }^{33}$ e de Sonogashira, ${ }^{34}$ e acoplamentos C-N e C-O vêm sendo desenvolvidas nos últimos 30 anos, e tornando-se cada vez mais versáteis e úteis, Figura $6{ }^{35,36}$

Os reagentes organometálicos de cobre (reagente de Gilman e seus derivados) estão entre os mais empregados para a formação de ligações C-C. ${ }^{37}$ A reação de cicloadição Huisgen tipo "Click" de acoplamento [3+2] de azidas e alcinos (CuAAC) ganhou versatilidade incrível a partir da interveniência da catálise de cobre, ${ }^{38}$ e hoje é empregada nas mais variadas áreas, especialmente no desenvolvimento de fármacos e compostos bioconjugados. ${ }^{39}$ Mais recentemente, a funcionalização $\mathrm{C}-\mathrm{H},{ }^{40}$ adições assimétricas conjugadas, ${ }^{41}$ adições assimétricas de acetiletos a compostos carbonílicos e imínios, ${ }^{42}$ sínteses heterocíclicas, ${ }^{43}$ reações CuAAC fotoativadas, ${ }^{44}$ dentre outras, têm mostrado a importância e o impacto que o cobre tem na química, e demonstrado seu papel central no desenvolvimento e aplicação de novas metodologias sintéticas.

A importância do uso das reações mediadas por cobre e seus sais é reconhecida por seu extenso uso por grupos nacionais em suas pesquisas e seus projetos. Várias sínteses totais e estudos metodológicos empregaram o metal em etapas importantes, e demonstram a versatilidade das reações citadas anteriormente. Um exemplo são os reagentes de Gilman, do tipo organocobre, e seus derivados, Esquema 1. A síntese da basiliskamida B utilizou metil-cianocuprato de lítio $[\mathrm{MeCu}(\mathrm{CN}) \mathrm{Li}]$ como agente alquilante para homologação e instalação do $\mathrm{C}_{12}$, através da formação da ligação C-C com o triflato intermediário. ${ }^{45}$ Já na síntese da macrolactina $\mathrm{A}$, empregou-se o cuprato misto de alta ordem $\mathrm{BuCu}(\mathrm{CN})(2-\mathrm{Th}) \mathrm{Li}_{2}$ para realizar a transmetalação com o Z-vinil-telureto preparado, e assim permitir a abertura do epóxido e formar o diol anti quiral. ${ }^{46}$

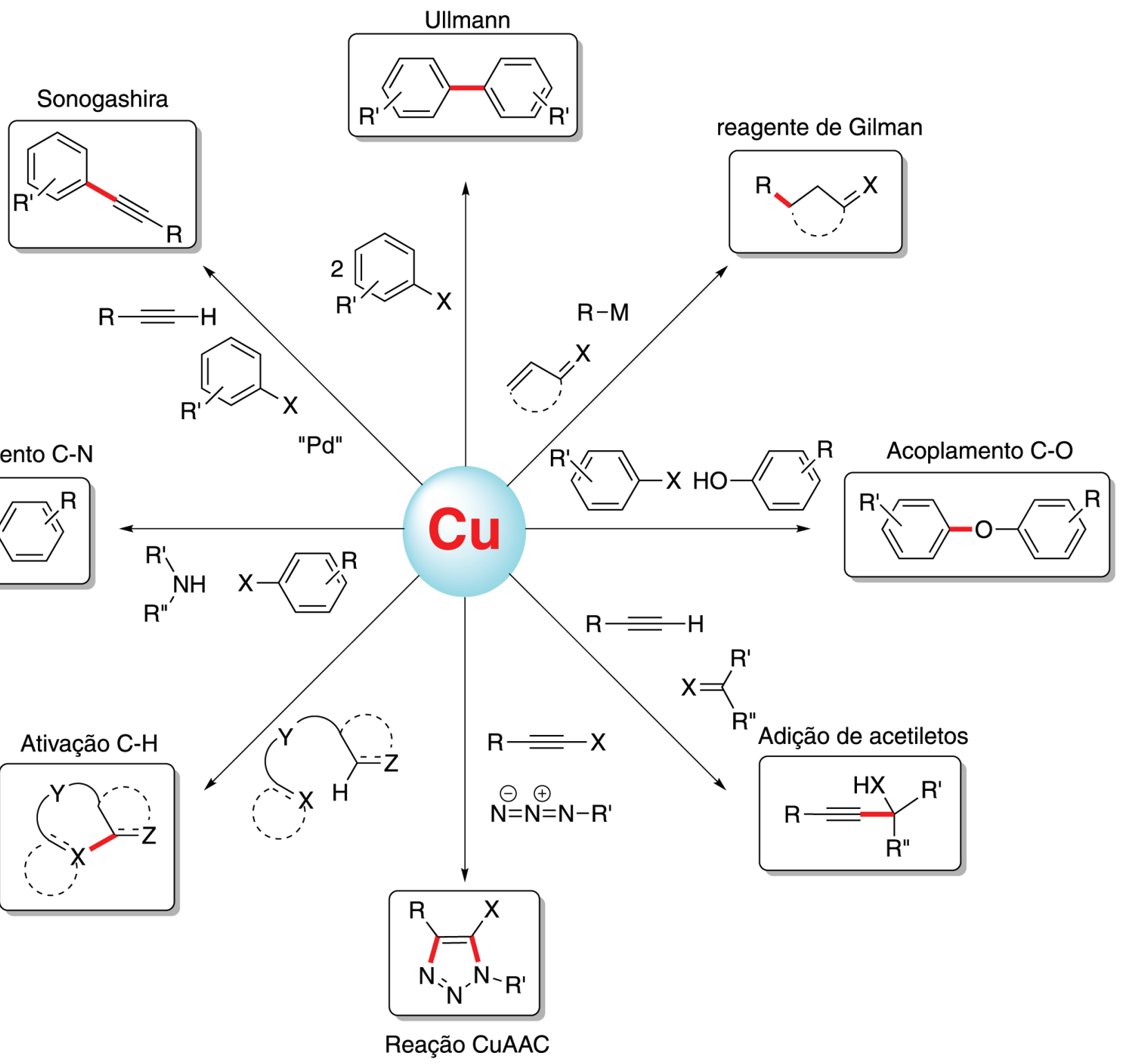

Figura 6. Principais reações orgânicas mediadas por cobre 


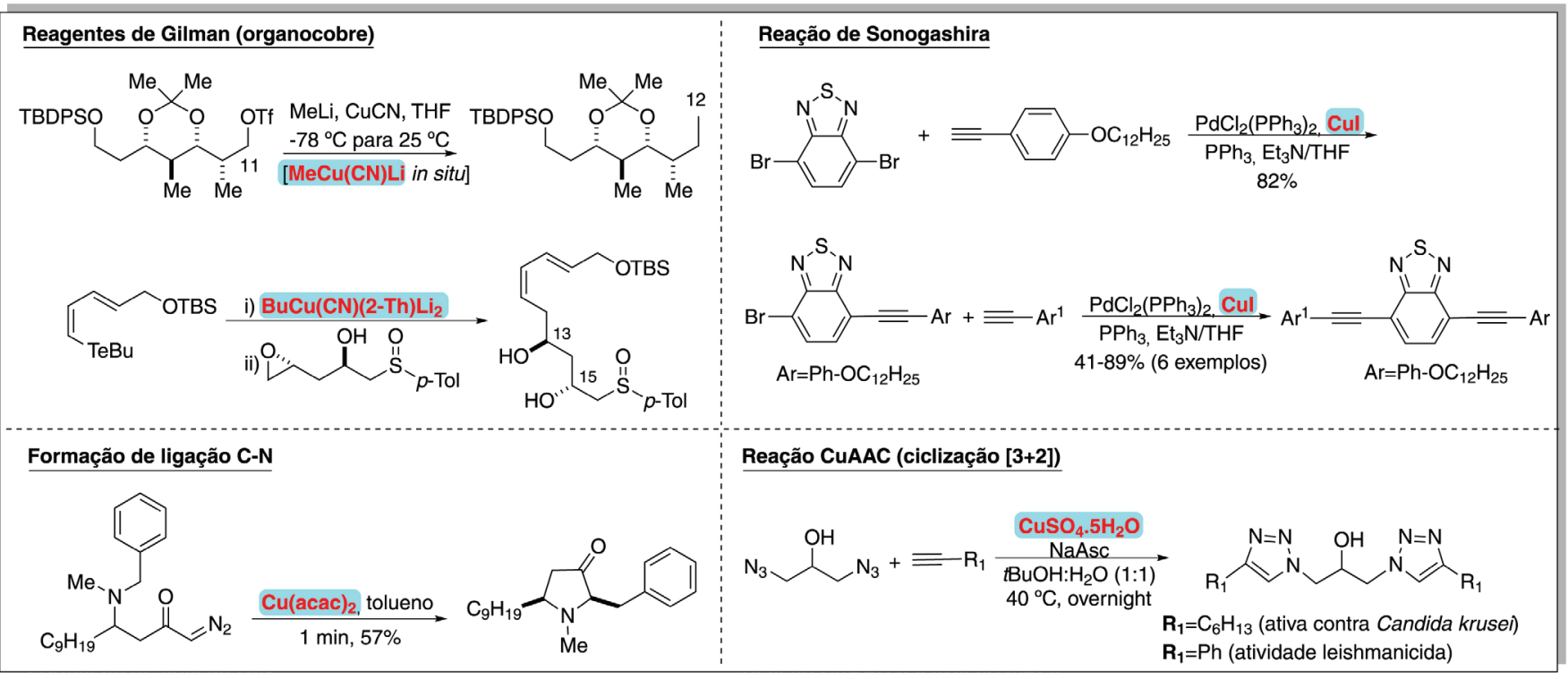

Esquema 1. Exemplos de reações orgânicas mediadas por reagentes de cobre

A fim de estudar o efeito de dissubstituição em cristais líquidos calamíticos, uma série de 2,1,3-benzotiadiazóis não-simétricos foi sintetizada, ${ }^{47}$ empregando-se a reação de Sonogashira como etapa-chave na formação de ligações C-C, Esquema 1. A preparação foi possível realizando-se duas etapas distintas e sequenciais de reação de acoplamento de alcinos terminais com o 4,7-dibromo-2,1,3-benzotiadiazol. Na primeira reação, o éter etinil-fenil-dodecílico foi empregado, e para a segunda reação de acoplamento de Sonogashira, uma série de diferentes alcinos terminais foram empregados, com o intuito de criar diversidade estrutural e assim estudar a influência sobre as propriedades ópticas dos derivados.

Estudos visando a síntese da preussina, através de rota bastante direta (apenas 3 etapas), empregaram a formação de ligação $\mathrm{C}-\mathrm{N}$ mediada por sal de cobre, Esquema 1. Adição de Michael da benzil-metilamina a uma diazocetona $\alpha, \beta$-insaturada forneceu o aduto aza-Michael intermediário. Esse foi, então, empregado em estudos de reação de formação de carbeno metálico, permitindo a formação da ligação C-N e, por consequência, do ilídio de amônio, seguido por um rearranjo de Stevens do tipo [1,2]. Os melhores resultados foram obtidos com a intervenção de $\mathrm{Cu}(\mathrm{acac})_{2}$ em tolueno. ${ }^{48}$

Sais de cobre são hoje largamente empregados nas reações do tipo click CuAAC, e são uma alternativa robusta na preparação de uma série de compostos com promissoras atividades biológicas. Empregando essa metodologia, uma série de bistriazóis foram preparados com o intuito de se avaliar suas atividades antifúngicas e leshmanicidas, Esquema 1. Os autores utilizaram 1,3-diazidas funcionalizadas ou não, e realizaram as reações de ciclização com alcinos terminais, oxigenados ou não, preparando uma série de bistriazóis. Apesar dos resultados com derivados dos alcinos oxigenados terem sido reduzidos, dois derivados obtidos mostraram atividade biológica importante: um contra uma cepa de fungos resistente ao fluconazol $\left(\mathrm{R}_{1}=\mathrm{C}_{6} \mathrm{H}_{13}\right)$, enquanto outro mostrou atividade inibitória leshmanicida na faixa micromolar $\left(\mathrm{R}_{1}=\mathrm{Ph}\right) .{ }^{49}$

\section{CONCLUSÃO}

O volume das reservas de minério de cobre que ocorrem no Brasil colocam esse elemento químico como uma importante comodity dentre os minérios exportados. Todavia, ainda se faz necessário investir tanto na ampliação da produção comercializada, que é apenas uma pequena fração da produção bruta nacional, quanto no beneficiamento para a produção do metal em alta pureza. Esses fatos se justificam em função das diversas aplicações do cobre metálico e seus derivados em áreas que abragem desde a geração e transmissão de energia elétrica e construção civil, até saúde, química inorgânica, química orgânica e bioquímica.

\section{AGRADECIMENTOS}

Os autores agradecem o suporte financeiro do Conselho Nacional de Desenvolvimento Científico e Tecnológico - CNPq e Fundação de Amparo à Pesquisa do Estado da Bahia - FAPESB. O presente trabalho foi realizado com apoio da Coordenação de Aperfeiçoamento de Pessoal de Nível Superior - Brasil (CAPES) - Código de Financiamento 001

\section{REFERÊNCIAS}

1. https://www.iypt2019.org/, acessado em outubro 2019.

2. Mendelejeff, D.; Z. Chem. 1869, 12, 405.

3. Dana, J.; Manual de Mineralogia, Editora Universidade de São Paulo, 1969.

4. Davenport, W. G.; Extractive Metallurgy of Copper, $4^{\text {th }}$ ed., Oxford, 2002.

5. Anuário Mineral Brasileiro, Principais Substâncias Metálicas, 2018, Ano Base 2017: http://www.anm.gov.br/dnpm/publicacoes/serie-estatisticase-economia-mineral/anuario-mineral/anuario-mineral-brasileiro/ amb_2018.pdf, acessado em outubro 2019

6. Greenwood, N. N.; Earnshaw, A.; Chemistry of the Elements; $2^{\text {nd }}$ ed., Butterworth Heinemann: Oxford, 1997.

7. Bohnet, M. Ullmann's Encyclopedia of Industrial Chemistry, $7^{\text {th }}$ ed. John Wiley, 2001.

8. https://www.paranapanema.com.br/show.aspx?idCanal=AEuEJAcpHV eaDfwBF2P2Nw==), acessado em outubro 2019.

9. Siotto, M.; Squitti, R.; Coord. Chem. Rev. 2018, 371, 86.

10. Gray, H. B.; Malmstrom, B. G., Williams, R. J. P.; J. Biol. Inorg. Chem. 2000, 5,551 .

11. Valensin, D.; Dell'Acqua, S.; Kozlowski, H.; Casella L.; J. Inorg. Biochem. 2016, 163, 292

12. Silva, L. A.; de Andrade, J. B.; J. Braz. Chem. Soc. 2004, 15, 170.

13. Chevreul, M. E.; Ann. Chim. 1812, 83, 187.

14. Silva, L. A.; de Andrade, J. B.; Toma, H. E.; J. Braz. Chem. Soc. 2002 , 13,624 . 
15. Fang, Y.; Guo, Y.; Chin. J. Catal. 2018, 39, 566.

16. Meng X. J.; Guo, H. Q.; Wang, Q.; Xiao, Y.; Chen, C. B.; Hou, B.; Li, D. B.; Catal. Sci. Technol. 2017, 7, 3511.

17. Ye, R.-P.; Lin L.; Li, Q.; Zhou, Z.; Wang, T.; Russell, C. K.; Adidharma, H.; Xu, Z.; Yao, Y.-G.; Fan, M.; Catal. Sci. Technol. 2018, 8, 3428.

18. Xie, H.; Wang, J.; Ithisuphalap, K.; Wu, G.; Li, Q.; Energy Convers. Manage. 2019, 188, 366.

19. Slamet; Nasution, H. W.; Purnama, E.; Riyani, K., Gunlazuardi, J.; World Appl. Sci. J. 2009, 6, 112.

20. Tseng, I. H.; Chang, W. C.; Wu, J. C. S.; Appl. Catal. B: Environ. 2002, 37,37

21. Adachi, K.; Ohta, K.; Mizuno, T.; Sol. Energy 1994, 53, 187.

22. Yamashita, H.; Nishiguchi, H.; Kamada, N.; Anpo, M.; Res. Chem. Intermed. 1994, 20, 815.

23. Park, H.; Ou, H-H.; Colussi, A. J.; Hoffmann, M. R.; J. Phys. Chem. A 2015, 119, 4658

24. Wang, G.; Ling, Y.; Wang, H.; Lu, X.; Li, Y.; Photochem. Photobiol. C 2014, 19, 35.

25. André, L.; Abanades, S.; Cassayre, L.; ACS Appl. Energy Mater. 2018, 1,3385 .

26. Wu, S.; Zhou, C.; Doroodchi, E.; Moghtaderi, B.; Energy Convers. Manage. 2019, 188, 366.

27. Reche, R. V.; Lima-Neto, A. F.; Silva, A. A.; Galinaro, C. A.; Osti, R. Z.; Franco, D. W.; J. Agric. Food Chem. 2007, 55, 6603.

28. Nascimento, R. F.; Cardoso, D. R.; Lima-Neto, B. S.; Franco, D. W.; Farias, J. B.; Quim. Nova 1998, 21, 735; Para aplicação de cachaça em aula ver: Cunha, S.; Matos, J. S.; Quim. Nova 2017, 40, 1253.

29. Cardoso, D. R.; Lima-Neto, B. S.; Franco, D.s W., Nascimento, R. F.; Quim. Nova 2003, 26, 165.

30. Patai, K.; Szilagyi, G.; Noszal, B.; Szentmariay, I.; Fertil. Steril. 2003, $80,1281$.
31. Chemler, S. R.; Beilstein J. Org. Chem. 2015, 11, 2252.

32. Ullmann, F.; Ber. 1903, 36, 2382.

33. Castro, C. E.; Stephens, R. D.; J. Org. Chem. 1963, 28, 2163.

34. Sonogashira, K.; Tohda, Y.; Hagihara, N.; Tetrahedron Lett. 1975, 4467.

35. Niu, J.; Zhou, H.; Li, Z.; Xu, J.; Hu, S.; J. Org. Chem. 2008, 73, 7814.

36. Zhang, Y.; Yang, X.; Yao, Q.; Ma, D.; Org. Lett. 2012, 14, 3056.

37. Hegedus, L. S.; Transition Metals in the Synthesis of Complex Organic Molecules, $2^{\text {nd }}$ ed., University Science Books: Sausalito, 1999.

38. Kolb, H. C.; Finn, M. G.; Sharpless, K. B.; Angew. Chem., Int. Ed. 2001, 40, 2004.

39. Meldal, M.; Tornoe, C. W.; Chem. Rev. 2008, 108, 2952.

40. Guo, X.-X.; Gu, D.-W.; Wu, Z.; Zhang, W.; Chem. Rev. 2015, 115, 1622.

41. Feringa, B. L.; Badorrey, R.; Pena, D.; Harutyunyan, S. R.; Minnaard, A. J.; Proc. Nat. Acad. Sci. 2004, 101, 5834.

42. Liu, J.; Dasgupta, S.; Watson, M. P.; Beilstein J. Org. Chem. 2015, 11, 2696.

43. Jeena, V.; Sithebe, S.; Robinson, R. S.; Synth. Commun. 2015, 45, 1484.

44. Alzahrani, A. A.; Nair, D. P.; Smits, D. J.; Saed, M.; Yakacki, C. M.; Bowman, C. N.; Chem. Mater. 2014, 26, 5303.

45. Dias, L. C.; Gonçalves, C. C. S.; Adv. Synth. Catal. 2008, 350, 1017.

46. Marino, J. P.; McClure, M. S.; Holub, D. P.; Comasseto, J. V.; Tucci, F. C.; J. Am. Chem. Soc. 2002, 124, 1664.

47. Benevides, T. O.; Regis, E.; Nicoleti, C. R.; Bechtold, I. H.; Vieira, A. A.; Dyes Pigments 2019, 163, 300.

48. Rosset, I. G.; Dias, R. M. P.; Pinho, V. D.; Burtoloso, A. C. B.; J. Org. Chem. 2014, 79, 6748.

49. Victor, M. M.; Farias, R. F.; Silva, D. L.; Carmo, P. H. F.; ResendeStoianoff, M. A.; Viegas, C.; Espuri, P. F.; Marques, M. J.; Med. Chem. 2019, 15, 400 . 